\title{
Transcriptomic Analysis of Oryza sativa Leaves Reveals Key Changes in Response to Magnaporthe oryzae MSP1
}

\author{
Qingfeng Meng ${ }^{1}$, Ravi Gupta ${ }^{1}$, Soon Jae Kwon', Yiming Wang ${ }^{2}$, Ganesh Kumar Agrawal ${ }^{3}$, \\ Randeep Rakwal ${ }^{3,4,5,6}$, Sang-Ryeol Park ${ }^{7}$, and Sun Tae Kim ${ }^{1 *}$ \\ ${ }^{\prime}$ Department of Plant Bioscience, Life and Energy Convergence Research Institute, Pusan National University, Miryang \\ 46241, Korea \\ ${ }^{2}$ Department of Plant-Microbe Interactions, Max Planck Institute for Plant Breeding Research, Carl-von-Linne Weg 10, \\ 50829 Cologne, Germany \\ ${ }^{3}$ Research Laboratory for Biotechnology and Biochemistry (RLABB), GPO Box 13265, Kathmandu, Nepal \\ ${ }^{4}$ GRADE Academy Private Limited, Adarsh Nagar-13, Birgunj, Nepal \\ ${ }^{5}$ Faculty of Health and Sport Sciences and Tsukuba International Academy for Sport Studies (TIAS), University of Tsu- \\ kuba, Ibaraki 305-8577, Japan \\ ${ }^{6}$ Global Research Center for Innovative Life Science, Peptide Drug Innovation, School of Pharmacy and Pharmaceuti- \\ cal Sciences, Hoshi University, Tokyo 142-8501, Japan \\ ${ }^{7}$ Gene Engineering Division, National Institute of Agricultural Sciences, Rural Development Administration, Jeonju \\ 54874, Korea
}

(Received on January 23, 2018; Revised on May 4, 2018; Accepted on May 14, 2018)

Rice blast disease, caused by Magnaporthe oryzae, results in an extensive loss of rice productivity. Previously, we identified a novel $M$. oryzae secreted protein, termed MSP1 which causes cell death and pathogen-associated molecular pattern (PAMP)-triggered immune (PTI) responses in rice. Here, we report the transcriptome profile of MSP1-induced response in rice, which led to the identification of 21,619 genes, among which 4,386 showed significant changes $(P<0.05$ and fold change $>2$ or $<1 / 2$ ) in response to exogenous MSP1 treatment. Functional annotation of differentially regulated genes showed that the suppressed genes were deeply associated with photosynthesis, secondary metabolism, lipid synthesis, and protein synthesis, while the induced genes were involved in lipid degradation, protein degradation, and signaling. Moreover, expression of genes

\footnotetext{
*Corresponding author.

Phone) +82-55-350-5505, FAX) +82-55-350-5509

E-mail) stkim71@pusan.ac.kr

(c) This is an Open Access article distributed under the terms of the Creative Commons Attribution Non-Commercial License (http:// creativecommons.org/licenses/by-nc/4.0) which permits unrestricted noncommercial use, distribution, and reproduction in any medium, provided the original work is properly cited.
}

Articles can be freely viewed online at www.ppjonline.org. encoding receptor-like kinases, MAPKs, WRKYs, hormone signaling proteins and pathogenesis-related (PR) proteins were also induced by MSP1. Mapping these differentially expressed genes onto various pathways revealed critical information about the MSP1-triggered responses, providing new insights into the molecular mechanism and components of MSP1-triggered PTI responses in rice.

Keywords : DNA microarray, Magnaporthe oryzae, MSP1, PAMP-triggered immunity, rice

Handling Associate Editor : Shim, Won Bo

As one of the most important food crops, rice feeds over $50 \%$ of the world's population. Rice blast, caused by Magnaporthe oryzae, is one of the most damaging diseases of rice that leads to yield loss of up to $30 \%$ in total rice production (Liu et al., 2014b; Talbot, 2003). Development of resistant cultivars is the best approach to control this disease, as this approach is cost effective and causes less environmental pollution (Dodds and Rathjen, 2010). During the course of evolution, plants have developed a two-layered immune system, consisting of i) a nonspecific and weak form via the recognition of pathogen-association molecular 
patterns (PAMPs), which leads to PAMP-triggered immunity (PTI) and ii) a highly specific and robust form via the recognition of pathogen effectors, known as effectortriggered immunity (ETI) (Boller and Felix, 2009; Boller and He, 2009; Jones and Dangl, 2006).

Cerato-platanin $(\mathrm{CP})$ proteins, which are fungal secreted proteins with four conserved cysteines residues, act as PAMPs to elicit defense responses and programed cell death (PCD) in hosts (Gaderer et al., 2014; Luti et al., 2016; Pazzagli et al., 2014). MSP1 (M. oryzae snodprot1 homolog protein), is one such protein which was first implicated in M. oryzae virulence, as MSP1 knock-out mutants showed reduced virulence (Jeong et al., 2007). Moreover, ectopic expression of MSP1 (also named as MoSM1) from $M$. oryzae has been shown to induce broad-spectrum disease resistance against Botrytis cinerea and Pseudomonas syringae pv. tomato in Arabidopsis (Yang et al., 2009). Recently, we reported that MSP1 induces autophagic PCD and elicits PTI in rice (Wang et al., 2016). Moreover, gene overexpression experiments revealed that MSP1 confers broad-spectrum resistance to rice blast and bacterial blight diseases (Hong et al., 2017). However, MSP1 response at the transcriptome level remains poorly understood.

Plant PTI can be activated when pattern recognition receptors PRRs, e.g. receptor like kinases (RLKs), recognize pathogens or elicitors (Boutrot and Zipfel, 2017). For instance, the plant membrane receptors FLS2 and CERK1 trigger PTI after recognizing bacterial and fungal elicitors flg22 and chitin, respectively (Akamatsu et al., 2013; Gómez-Gómez and Boller, 2000). Following PRRs recognition, activated signaling is transduced via MAPK signaling cascades from PRRs to multiple cellular defense responses, including the biosynthesis/signaling of plant defense hormones, reactive oxygen species (ROS) generation, defense genes activation (Meng and Zhang, 2013). In rice, OsRLCK185 mediates the chitin-triggered PTI directly from OsCERK1, and the signaling is translocated via MAPK cascades (Wang et al., 2017). The Arabidopsis protein PBL27 mediates chitin-triggered immunity from AtCERK1 to MAPK cascades (Yamada et al., 2016). As one of the downstream targets of MAP kinases, WRKY transcription factors (TFs) regulate plant defense signaling during defense gene transcription processes (Eulgem and Somssich, 2007). For instance, Arabidopsis MPK3 directly phosphorylates the WRKY33, which is essential for immune responses against bacterial and necrotrophic fungal pathogens (Eckardt, 2011).

Although, numerous transcriptional analyses related to the rice- $M$. oryzae interaction have been reported leading to identification of several components of $M$. oryzae trig- gered PTI signaling (Bagnaresi et al., 2012; Wang et al., 2014; Wei et al., 2013), no studies have been conducted to identify the components of MSP1-induced PTI signaling. Therefore, in this study a DNA microarray analysis was conducted to identify transcriptional changes in response to exogenous MSP1 treatments in rice, for the first time to the best of our knowledge. Potential genes involved in physiological reactions downstream of MSP1-induced PTI were identified via transcriptome comparison, including RLKs, MAPKs, WRKYs, PRs and hormone signaling related genes.

\section{Materials and Methods}

Plant material and growth conditions. Rice seeds (Oryza sativa L. Dongjin) were sterilized in $0.05 \%$ Spotak solution (Bayer crop science, South Korea) overnight at $4^{\circ} \mathrm{C}$, and washed with distilled water 5 times. Sterilized seeds were then placed onto moist tissue paper and incubated at $28^{\circ} \mathrm{C}$ in the dark for germination. Germinated seeds were transferred to sterilized soil in a growth chamber (70\% humidity, $25^{\circ} \mathrm{C}$, and a light and dark cycle of 14 and $10 \mathrm{~h}$ ) for four weeks.

Sample preparation. For DNA microarray analysis, fourweek-old rice leaves were sprayed with $5 \mathrm{ml}$ of solution containing PBS (control) or $1 \mu \mathrm{M}$ of purified recombinant MSP1-His protein dissolved in PBS, using a foliar spray. Recombinant MSP1-His protein was expressed in E. coli, and purified as previously described (Wang et al., 2016). Each treatment was performed using six plants in two replicates $(6 \times 2$ rice plants/treatment), resulting in 12 plants per treatment. Treated leaves were harvested and frozen in liquid nitrogen at $6 \mathrm{~h}$ post-treatment (hpt) and $24 \mathrm{hpt}$, then stored at $-70^{\circ} \mathrm{C}$ until further analysis. A total of six RNA samples were subjected to DNA microarray analysis.

DNA microarray. Total RNA was isolated using an RNeasy Plant Mini Kit (QIAGEN, Hilden, Germany) according to the manufacturer's instructions. Genomics DNA was removed with DNase I (QIAGEN, Hilden, Germany). Synthesis of target cRNA was performed using Agilent's Low RNA Input Linear Amplification Kit PLUS (Agilent Technology, CA, USA) according to the manufacturer's instructions. Briefly, each $0.2 \mu \mathrm{g}$ of total RNA was mixed with the diluted Spike mix and T7 promoter primer mix, then incubated at $65^{\circ} \mathrm{C}$ for $10 \mathrm{~min}$. Next, cDNA master mix (5× First strand buffer, $0.1 \mathrm{M}$ DTT, $10 \mathrm{mM}$ dNTP mix, RNase-Out, and MMLV-RT) was prepared and added to the reaction mixer. The procedures for DNA microar- 
ray were identical to those described in our previous study (Wang et al., 2014). All data normalization and selection of fold-changed genes was performed using GeneSpring GX 7.3 (Agilent Technology). Genes were filtered by removing flag-out genes in each experiment. The averages of normalized ratios were calculated by dividing the test channel intensity by the control channel intensity.

Bioinformatics analysis. The MapMan program, version 3.6.ORC1 (Thimm et al., 2004), was used for pathway analysis. Fold-change values were $\log _{2}$ transformed, after which their means were calculated. These genes were classified into MapMan BINs and their annotated functions were visualized using the MapMan program by searching against Oryzae sativa Osa_MSU_v7 mapping. The PageMan visualization tool was used for GSEA with the Wilcoxon test (Benjamini_Hochberg correction and 1.0 as the ORA cutoff).

The pathway enrichment study was performed using the KEGG database (Kanehisa et al., 2014) with the DAVID Functional Annotation Tool against the background of Oryza sativa (Huang et al., 2008). Genes up-regulated or down-regulated in MSP1 treated samples at each sampling time point were loaded as gene lists in DAVID. A cor- rected $\mathrm{p}$-value $<0.05$ denoted the significance of enriched pathways. The top 10 significantly enriched pathways of each list were selected for comparison.

Validation of DNA microarray analysis by qPCR. Two ug (this should be greek symbol micro) of total RNA from each treated sample (the same samples as for the microarray) was used to synthesize cDNA with RNA to cDNA EcoDry Premix kit (Clontech, Mountain View, CA, USA). qPCR was performed using a Rotor-Gene Q instrument (QIAGEN, Hilden, Germany) with QuantiNova SYBR Green RT-PCR kit (QIAGEN, Hilden, Germany). The following PCR reaction program was used: $95^{\circ} \mathrm{C}$ for $10 \mathrm{~min}$ followed by 40 cycles of $95^{\circ} \mathrm{C}$ for $10 \mathrm{~s}$ and $60^{\circ} \mathrm{C}$ for $20 \mathrm{~s}$. Fold change was calculated relative to plants treated with PBS. The transcript level represented the mean of three replicates after normalization using Ubiquitin 5 (LOC_ Os01g22490.1), which varied little in our microarray analysis and was previously used as a reference gene (Jain et al., 2006). Primers used are listed in Supplementary Table 1.

\section{Results}

Dynamic transcriptomics changes in response to MSP1

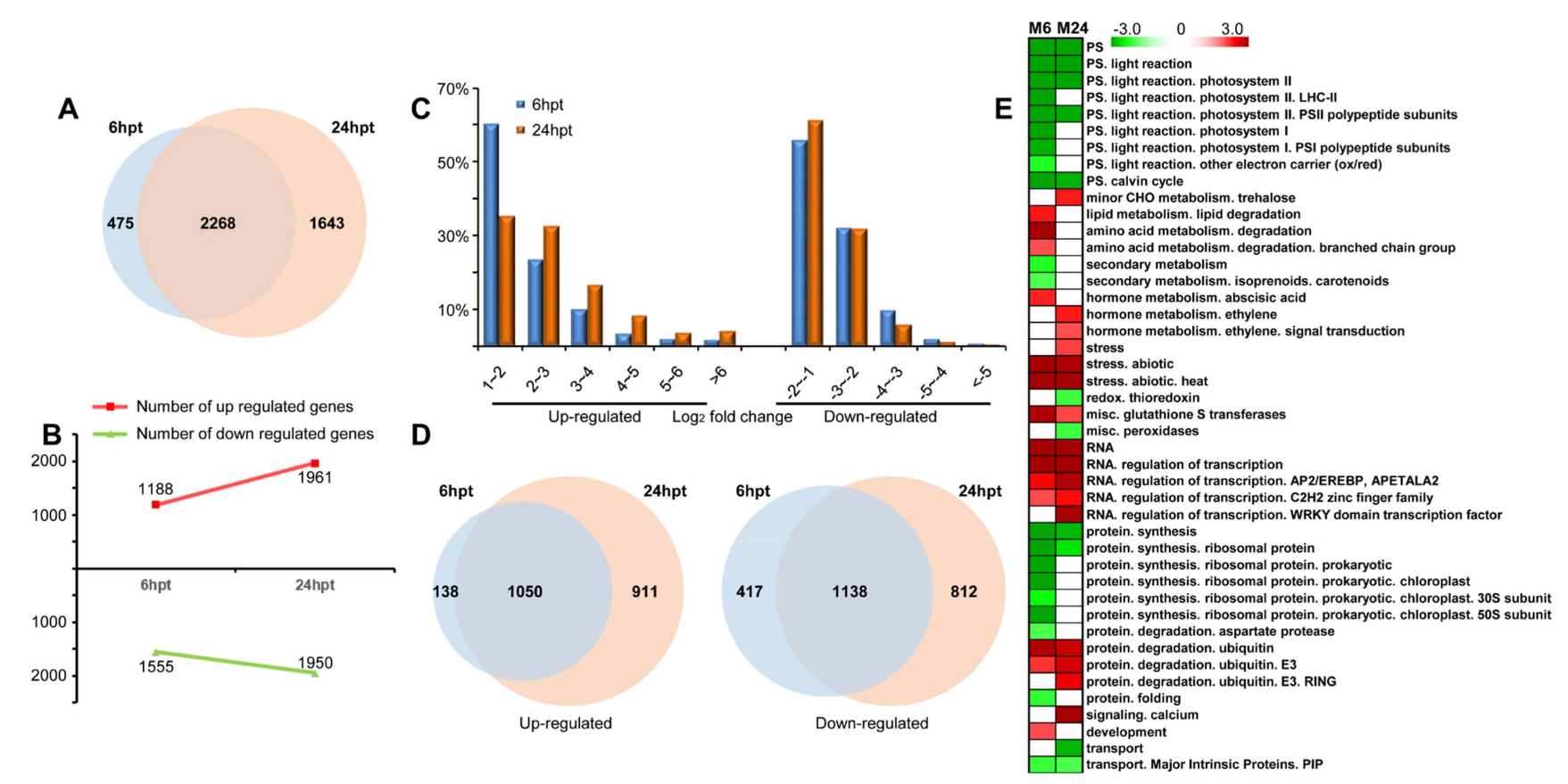

Fig. 1. Distribution of differentially expressed genes (DEGs) in response to MSP1 in rice leaves. M6, MSP1 treated sample harvested at $6 \mathrm{hpt}$; M24, MSP1 treated sample harvested at $24 \mathrm{hpt}$. (A) Diagrams showing an overview of DEGs in response to MSP1 at 6 hpt and 24 hpt. (B) Numbers of up-regulated and down-regulated DEGs in response to MSP1. (C) Numbers of DEGs with different fold change range. (D) Diagram showing distribution of significantly up-regulated genes and down-regulated genes at $6 \mathrm{hpt}$ and $24 \mathrm{hpt}$. (E) Gene set enrichment analysis using the Pageman web-tool showed up- and down-regulated pathways at transcriptomics level in response to MSP1 treatment. A full list of data is given in supplementary Table 2. 
treatment. DNA microarray analysis was used to investigate differentially expressed genes (DEGs) ( $p$ value $<$ 0.01 and $\log _{2}$ fold change $>1$ or $<-1$ ) in response to MSP1 treatment in rice (Fig. 1, Supplementary Table 2). A total of 2268 DEGs were identified at both time points, whereas 475 and 1643 DEGs were up-regulated only at $6 \mathrm{hpt}$ and $24 \mathrm{hpt}$, respectively (Fig. 1A), indicating a high similarity of DEGs at different sampling time points. These results indicate that MSP1 induces a dramatic change in rice at the transcriptional level. Among all DEGs, 1188 were upregulated and 1555 were down-regulated at $6 \mathrm{hpt}$, while 1961 genes were up-regulated and 1950 genes were downregulated at $24 \mathrm{hpt}$ (Fig. 1B). Interestingly, almost $60 \%$ of
DEGs showed less than 4-fold changes ( $\log _{2}$ fold change $<2)$ at $6 \mathrm{hpt}$ and the percentage of DEGs with higher fold changes $\left(\log _{2}\right.$ fold change $\left.>2\right)$ were increased in $24 \mathrm{hpt}$ relative to the $6 \mathrm{hpt}$ samples (Fig. 1C), indicating that at 24 hpt rice exhibits a more intense response to MSP1 treatments. Among all up-regulated genes, 1050 were present in both samples, with 138 and 911 genes specific for $6 \mathrm{hpt}$ and $24 \mathrm{hpt}$, respectively (Fig. 1D). Among down-regulated genes, 1138 were present in both samples, with 417 and 812 genes specific for $6 \mathrm{hpt}$ samples and $24 \mathrm{hpt}$ samples, respectively (Fig. 1D). These results indicate that the transcriptomic changes in response to MSP1 occurred more dramatically at $24 \mathrm{hpt}$.

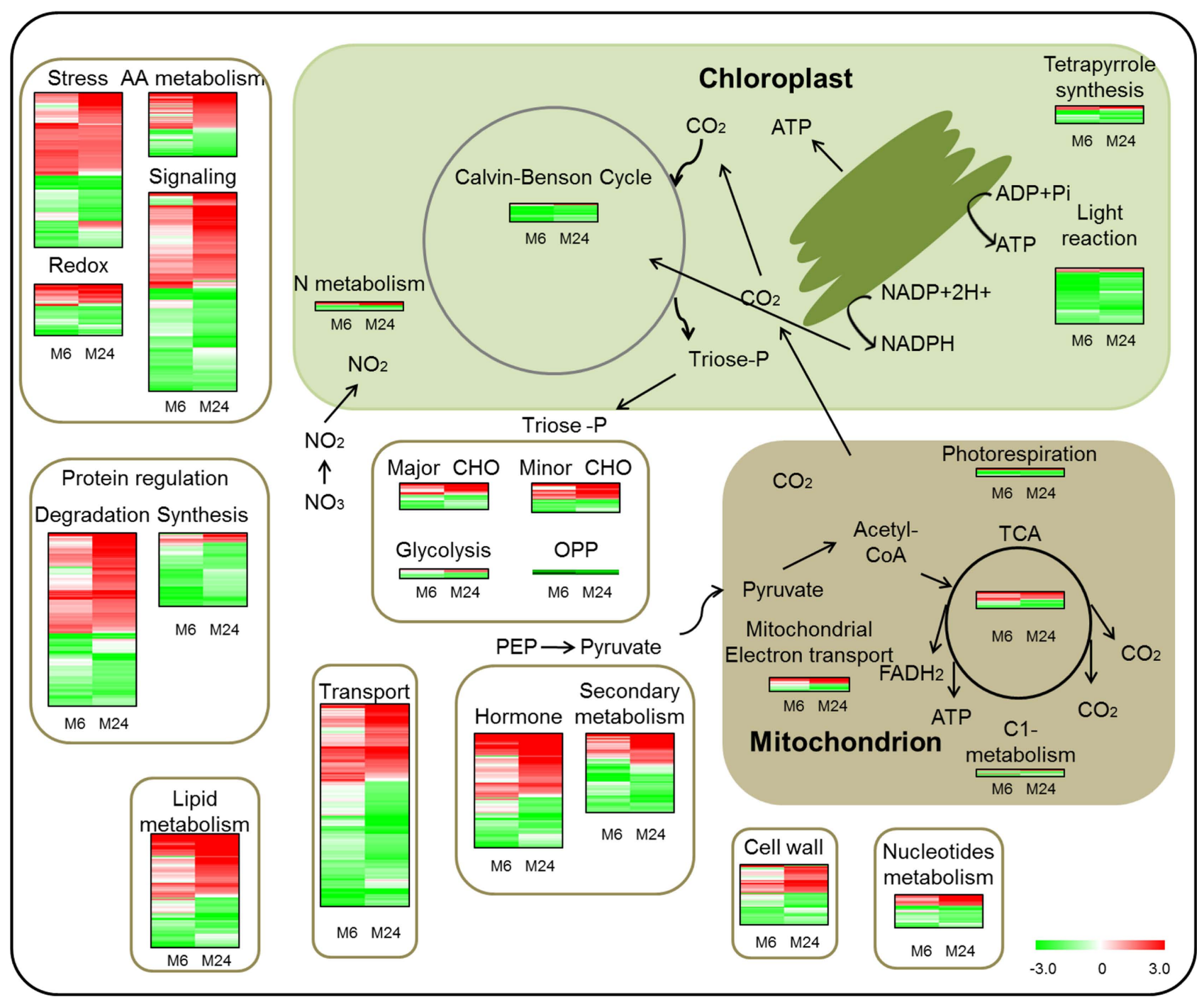

Fig. 2. Functional overview of genes significantly changed in different metabolic pathways represented as heat maps. The Mapman software was used to conduct functional categorization. M6, MSP1 treated sample harvested at 6 hpt; M24, MSP1 treated sample harvested at $24 \mathrm{hpt}$. Red indicates higher and green indicates lower expression in MSP1 treated sample, respectively. A full list of data and statistics are given in supplementary Table 3 . 
Gene set and pathway enrichment analysis. The PageMan web tool of MapMan was used for functional annotation of the DEGs. Both $6 \mathrm{hpt}$ and $24 \mathrm{hpt}$ samples exhibited an elevation in glutathione $\mathrm{S}$ transferases, abiotic stress, regulation of transcription and protein degradation, while down-regulated genes were related to photosynthesis, protein synthesis and transport (Fig. 1E, Fig. 2, Supplementary Table 3). At $6 \mathrm{hpt}$, genes related to lipid degradation, amino acid degradation, abscisic acid metabolism, and development were up-regulated, while genes associated with secondary metabolism, ribosomal protein in protein synthesis, aspartate protease and protein folding were downregulated (Fig. 1E, Fig. 2, Supplementary Table 3). At $24 \mathrm{hpt}$, up-regulated genes were mainly involved in minor CHO metabolism, ethylene metabolism, WRKY domain containing transcription factor and calcium signaling, while down-regulated genes were involved in processes of redox signaling (thioredoxin) and peroxidases (Fig. 1E, Fig. 2, Supplementary Table 3). These results show the processes responsive to MSP1 induced signaling at different sampling time points.

KEGG pathway enrichment analysis using DAVID bio- informatics tool helped to determine the metabolic pathways that were commonly or specifically affected by MSP1. Alpha-linolenic acid metabolism, glutathione metabolism and branched chain amino acid degradation (valine, leucine, and isoleucine) were commonly up-regulated, while photosynthesis, photosynthesis-antenna proteins, carbon fixation in photosynthetic organisms, glyoxylate and dicarboxylate metabolism and biosynthesis of antibiotics were commonly down-regulated at both $6 \mathrm{hpt}$ and $24 \mathrm{hpt}$ in response to MSP1. Furthermore, at $6 \mathrm{hpt}$, protein processing in the endoplasmic reticulum, nitrogen metabolism, starch and sucrose metabolism and tryptophan metabolism were up-regulated, while the pentose phosphate pathway and ribosome were specifically inhibited (Fig. 3A, B). At $24 \mathrm{hpt}$, genes related to plant hormone signal transduction, plantpathogen interaction, galactose metabolism, amino acid metabolism (alanine, aspartate, and glutamate) and inositol phosphate metabolism were enhanced, while porphyrin and chlorophyll metabolism and amino acid metabolism (glycine, serine, and threonine) were repressed (Fig. 3C, D).

Biotic stress-related pathway in response to MSP1

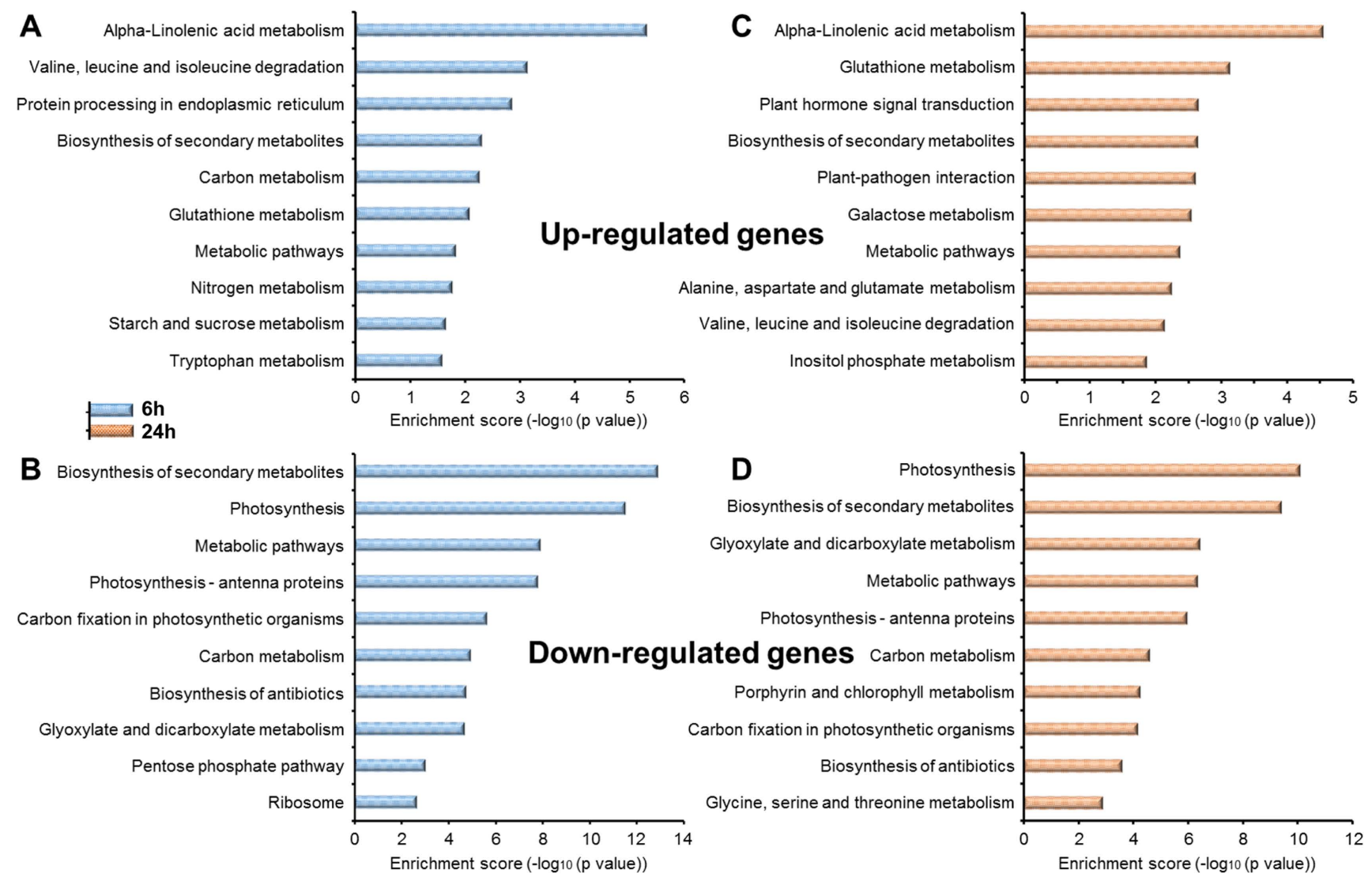

Fig. 3. Pathway enrichment of DEGs identified in MSP1 samples at 6 hpt and 24 hpt. (A-B) Significantly enriched pathway identified in MSP1 treated samples at the $6 \mathrm{hpt}$. (C-D) Significantly enriched pathway in the $24 \mathrm{hpt}$ samples. 
treatment. The expression pattern and functions of differentially regulated genes were visualized using MapMan to get insights into their biotic stress-related functions. Of the total 4836 DEGs, 1106 (22.8\%) were classified as biotic stress response genes (Fig. 4, Supplementary Table 4). Functional classification of biotic stress response genes indicated a general elevation of ABA metabolism, ethylene metabolism, JA metabolism, signaling, genes encoding glutathione-S-transferases, heat shock proteins, $\beta$-glucanase, transcription factors (ERF, bZIP, WRKY, MYB, DOF) and genes involved in proteolysis. In cases of hormone signaling, 14 of 15 genes (e.g., NCED2, NCED3, BZIP23, aldehyde oxidase $A O$ and carotenoid cleavage dioxygenase $8 a$ ) involved in ABA, 30 of 46 genes (e.g., ethylene response factor ERF3, ERF57, ERF77, ERF83, ERF91, ERF95, ERF96 and ERF98, OsACS2 and OsbHLH060) involved in ethylene and 11 of 16 genes (e.g., allene oxide synthase AOS2, AOS3, lipoxygenase LOX1, LOX6, LOX8, 12-oxo-phytodienoic acid reductase OPR1, OPR5, OPR6, OPR 9 and OsJAZ5) involved in JA signaling were induced by MSP1 treatment. Thirty of 43 genes encoding heat shock proteins (including HSP90, HSP82, HSP70, HSP 26, HSP24.1, HSP18.0, HSP17.9, HSP 17.7, HSP17.3 and HSP 16.9) were up-regulated in response to MSP1 treatment. Moreover, 26 of 33 genes encoding glutathione -S- transferases (e.g., GSTF10, GSTF11, GSTF5, GSTL2, GSTL3, GSTT1, GSTU1, GSTU12, GSTU19, GSTU21, GSTU24, GSTU28, GSTU31, GSTU37, GSTU40, GSTU47, GSTU50, GSTU7, GSTU8 and GSTU9) were induced by MSP1 treatment. These results indicate that MSP1 treatments induce a dramatic defense response in rice leaves.

Receptor-like kinases. In plants, receptor-like kinases (RLKs) and receptor-like proteins (RLPs) are employed as PRRs to monitor the environment and detect potential dangers (Boutrot and Zipfel, 2017). The RLKs are a large protein family with over 600 members in the model plant Arabidopsis thaliana, which have 1131 members in rice (Shiu et al., 2004). Of 89 RLK genes differentially regulated in MSP1 treated samples, 31 were up-regulated in at least in $24 \mathrm{hpt}$ samples. Most of these 31 RLKs were leucine-rich repeat RLKs and S-locus glycoprotein like RLKs. When

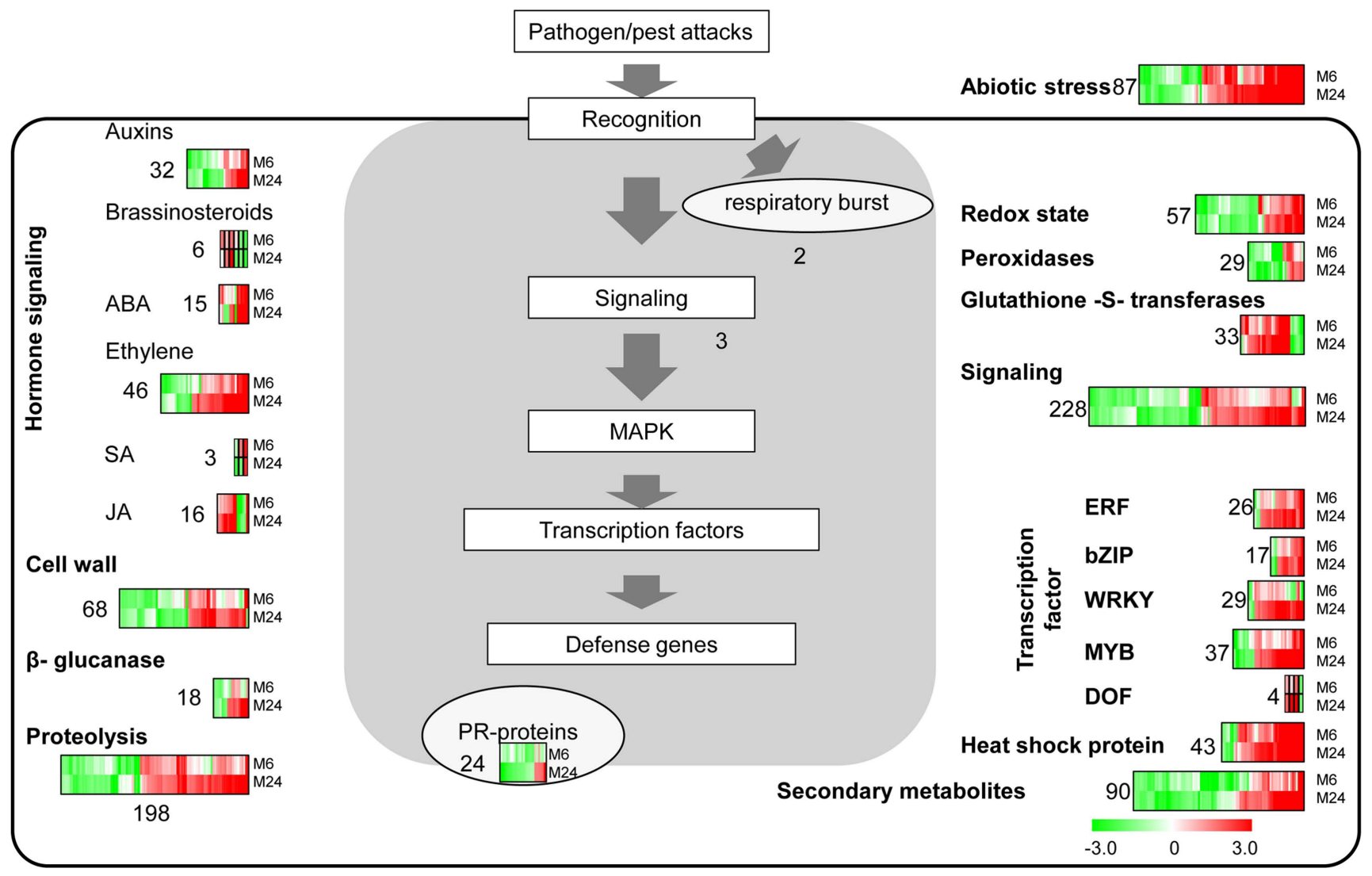

Fig. 4. Biotic stress-related genes altered in response to MSP1 treatment. Genes that were differentially regulated between control and MSP1 treatment are indicated by colors. M6, MSP1 treated sample harvested at $6 \mathrm{hpt}$; M24, MSP1 treated sample harvested at 24 hpt. A full list of data is provided in supplementary Table 4. 
compared with transcriptomics changes in $M$. oryzae ( $\mathrm{R}$ line, KJ401; S line, KJ301) infected rice leaves we reported previously (Wang et al., 2014), these RLKs showed a high similarity of expression patterns in MSP1 treated samples (Fig. 5B), demonstrating their importance in rice immune responses.

Signal transduction by the MAPK cascades. MAPK (mitogen-activated protein kinase) cascades, a set of kinases for signal transduction through phosphorylation, were activated as one of the earliest signaling events after recognition of PAMPs by plant PRRs, which consist of a MAPKKK-MEK-MPK module (Meng and Zhang, 2013). To identify important MAPK signaling cascades responsive to MSP1, we focused on expression changes in all MAPKKK, MEK and MPK genes. In total, 59 MAPKKK, four MEK, and 12 MPK genes were differentially regulated in MSP1 treated samples. Of these MAPK genes, 14 MAPKKK, one MEK, and two MPK genes were induced at $24 \mathrm{hpt}$ (Fig. 5A). Among the induced genes, 13 MAPKKK genes, one MKK gene and one MPK gene were also up-regulated at $6 \mathrm{hpt}$. In $24 \mathrm{hpt}$ samples, the most strongly induced MAPK gene was MAPKKK62, which was also highly up-regulated in $6 \mathrm{hpt}$ samples. Comparison of MAPK genes between MSP1 treated samples and M. oryzae infected samples revealed a high similarity of expression patterns.

WRKY transcription factors. WRKY transcription factors were reported to regulate the transcriptional reprogramming related to plant immune responses (Eulgem and Somssich, 2007). The rice genome contains a total of 113 WRKY genes, which is greater than the number of WRKY genes in Arabidopsis (Ryu et al., 2006). In total, 55 differentially regulated WRKY genes were identified in MSP1 treated samples. Of these, 30 were up-regulated in the 24 hpt samples and selected for comparison between MSP1 treated sample and M. oryzae infected samples, revealing a high similarity of expression patterns (Fig. 5A, Supplementary Table 5).

Genes encoding the pathogenesis-related (PR) proteins. Pathogenesis-related proteins have been reported to possess antimicrobial activities via hydrolytic activities on cell
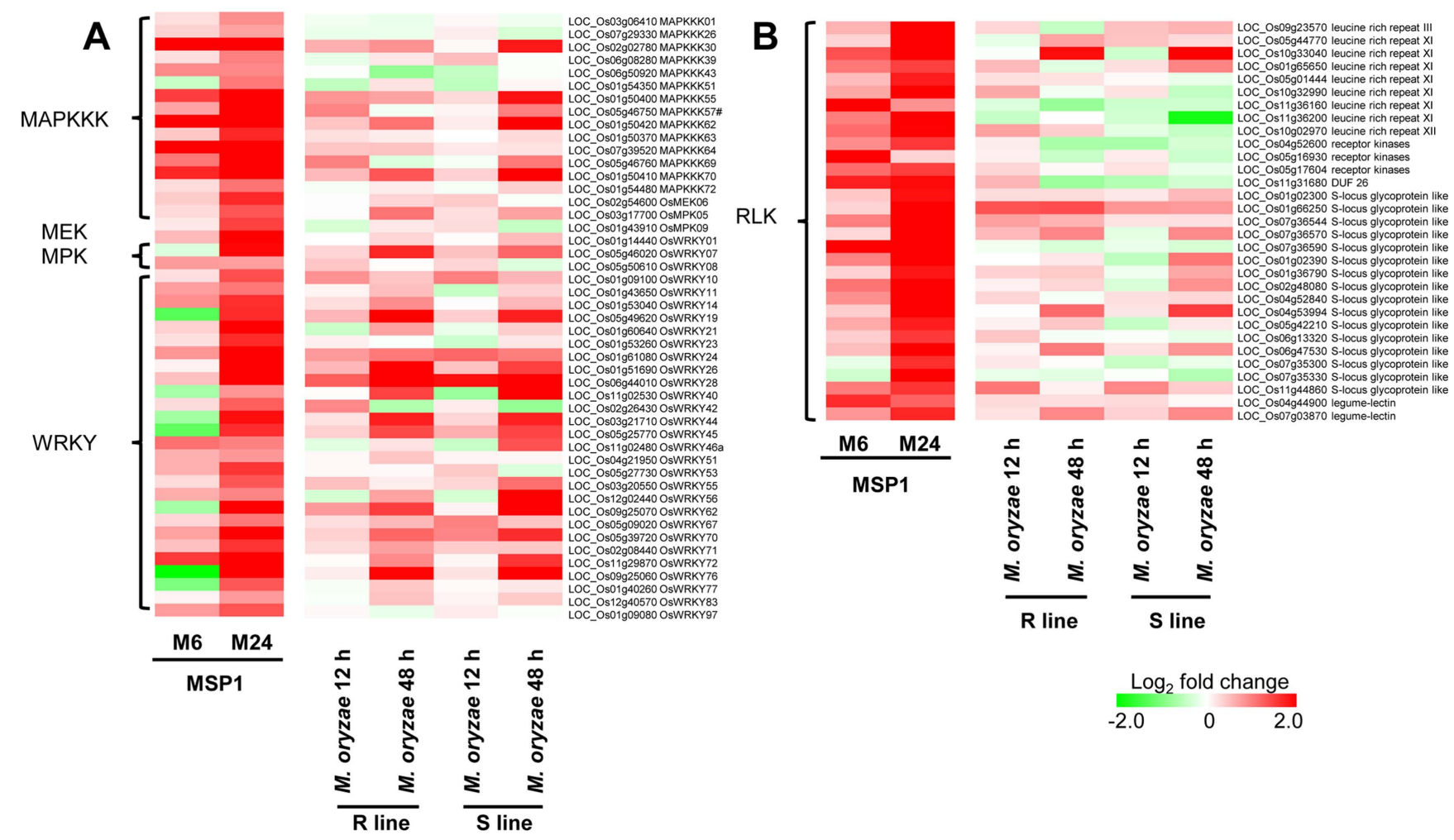

Fig. 5. RLKs, MAPKs and WRKY in response to MSP1 treatment and M. oryzae infection. (A) MAPKs and WRKYs; (B) Receptor like kinases (RLKs). A full list of the data is given in supplementary Table 5. M6, M24, rice samples harvested at 6 and $24 \mathrm{~h}$ after MSP1 treatment, respectively. $\mathrm{R}$ line, $\mathrm{S}$ line, rice leaf samples inoculated with a conidial suspension $\left(1 \times 10^{5}\right.$ conidia $\left./ \mathrm{ml}\right)$ of $M$. oryzae race $\mathrm{KJ} 401$ (incompatible to rice) and KJ301 (compatible to rice), respectively. 


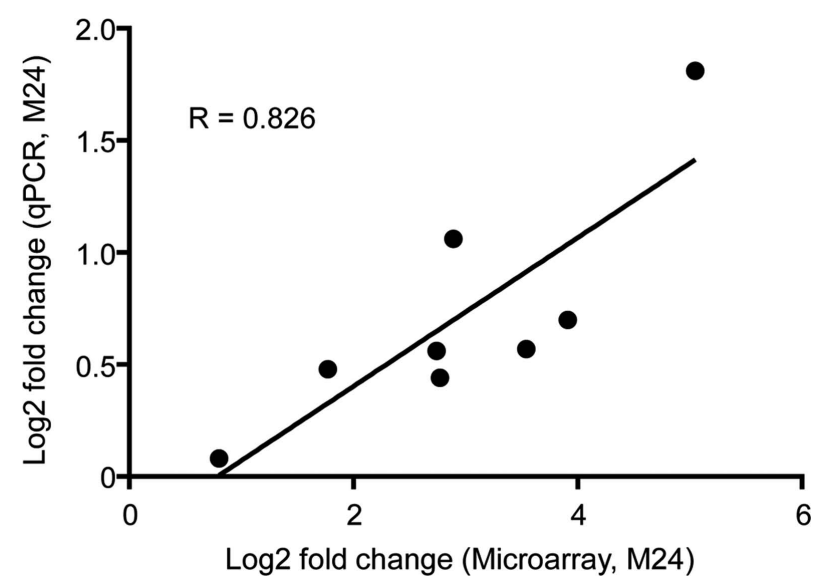

Fig. 6. Correlation of gene expression level by qPCR and microarray. The $\log 2$ value of the expression level (MSP1/con) (y-axis) from qPCR was plotted against the microarray value (x-axis). M24, samples harvested at $24 \mathrm{hpt}$. All qPCR data were obtained from three biological replicates and three technical replicates for each sample.

walls, contact toxicity, and involvement in defense signaling (van Loon et al., 2006). In total, 19 genes encoding PR proteins were detected in response to MSP1 treatment. Among these, three (JIOSPR10, CHT1, and PR1-71) were commonly induced in samples at both time points, while five genes (PR1OB, PR4C, CHT3, PR5 and PR1b) and one genes (PRla) were specifically up-regulated in the 24 and $6 \mathrm{hpt}$ samples, respectively. These results indicate that MSP1 treatment induces an intensive defense response in rice leaves at both time points.

Validation by qPCR. To validate the expression patterns of the identified DEGs, eight were further selected and confirmed by qPCR in $24 \mathrm{hpt}$ samples. Relative expression levels obtained by qPCR were converted to $\log _{2}$ fold change (MSP1/PBS treatment) for comparison with microarray data. Analysis of these eight genes using both techniques showed a high correlation $(\mathrm{R}=0.826)$ in $24 \mathrm{hpt}$ samples, indicating the high fidelity of the obtained data (Fig. 6, Supplementary Table 6).

\section{Discussion}

In this study, we employed a DNA-microarray-based transcriptomic approach to investigate the gene regulation in response to $M$. oryzae MSP1 protein. Rice leaves were treated with PBS (control) and MSP1 recombinant protein and harvested at $6 \mathrm{hpt}$ and $24 \mathrm{hpt}$. A total of 4386 DEGs were detected in response to MSP1 treatment. Further bioinformatics analyses, including functional data mining and pathway participation, were employed to identify specific key transcriptional changes in response to MSP1 treatment.

MSP1 downregulates photosynthesis and plant shifts to non-assimilatory metabolism. During pathogen attack, recognition of pathogens or elicitors leads to a massive reprogramming of plant cells to activate and deploy defense responses (Bolton, 2009; Rojas et al., 2014). Assignment of recourses for immune response and biosynthesis of protective compounds leads to an increased demand for energy and carbon skeleton, which are supported by primary metabolic pathways (Bolton, 2009; Rojas et al., 2014).

Numerous studies have reported that photosynthesis is impaired when plants shift towards non-assimilatory metabolism in response to pathogen attack (Kangasjärvi et al., 2012; Major et al., 2010; Roberts and Paul, 2006). In the present study, 59 of 63 genes involved in light reaction of photosynthesis, 18 of 19 Calvin cycle genes and 14 of 18 tetrapyrrole biosynthesis genes were down-regulated by MSP1 treatment (Fig. 2, 3, Supplementary Table 3), indicating broad down-regulation of photosynthesis in response to MSP1 treatment. These results are in line with those of previous reports that biotic stress causes uniform down regulation of the transcription of genes encoding the major components of photosynthesis (Bilgin et al., 2010). Thirty of 45 genes (e.g., fatty-acid desaturase OSSSI2, Acyl-CoAbinding protein $O s A C B P 1$ and $O s L A C S 1$ ) involved in lipid synthesis and 73 of 83 genes related to protein synthesis were down-regulated in MSP1 treated samples, suggesting the activation of non-assimilatory metabolism in response to MSP1 treatment. Previously, it was observed that OsSSI2-knock down plants exhibit markedly enhanced resistance to blast and leaf blight diseases in rice and Arabidopsis (Liu et al., 2014a; Shah et al., 2001), indicating it is a negative regulator of stress tolerance in plants. Here, we observed down-regulation of OSSSI2 in response to MSP1 treatments, indicating an enhanced resistance by MSP1.

Inhibition of photosynthesis activity and collapse of assimilatory metabolism results in a metabolic transition in which the demand for carbohydrates and energy is achieved through increased activities of respiratory metabolism and other types of metabolism (SmedegaardPetersen and Stolen, 1981). In the present study, three main pathways of respiration were activated during the resistance response, including glycolysis, the TCA cycle, and the electron transport chain. Three of eight genes encoding glycolysis-related proteins, ten of 18 genes involved in the TCA cycle, and seven of 16 genes involved in mitochondrial electron transport were up-regulated by MSP1 treatment, revealing a transcriptional change in the three main 
pathways of respiration in response to MSP1. Totally, 23 of 28 genes involved in lipid degradation and 115 of 198 genes involved in protein degradation were up-regulated by MSP1 treatment, providing an alternative respiratory substrate for MSP1-treated rice. Moreover, the up-regulation of protein and lipid degradation indicates that senescence processes in rice leaves may be highly elevated and nutrients released are mobilized from the leaves to other parts of the plants to support the immune response.

Defense signal transduction pathways were activated by MSP1 treatments. PTI is a form of basal defense that confers resistance to broad-spectrum pathogens in plants (Boller and Felix, 2009; Niks et al., 2015). Pattern recognition receptors (PRRs) trigger PTI, a relatively weak immune response restricting pathogen growth by recognizing highly conserved PAMPs. In this study, most of the 31 up-regulated RLK genes were leucine-rich repeat (LRR) RLKs and S-locus glycoprotein like RLKs, indicating potential RLKs for MSP1 triggered immunity. In FLS2, EFR, and Xa21, there is a large ectodomain including 28 LRRs, 21 LRRs, and 23 LRRs, respectively (Gómez-Gómez and Boller, 2000; Song et al., 1995; Zipfel et al., 2006), which are directly involved in elicitor binding (Antolín-Llovera et al., 2012). The DUF26 RLKs, which are cysteine-rich RLKs, comprise a large subgroup of RLKs involved in plant defense and programmed cell death (Chen et al., 2004). In the present study, the gene encoding OsDUF26 (also named $O S R L K$ ) was highly up-regulated by MSP1 treatment, which is in agreement with the results of a previous study in which OsDUF26 was induced by M. oryzae infection and fungal elicitor at both the RNA and protein levels (Kim et al., 2004, 2009b).

Following RLKs recognition, MAPK cascades are activated. In the present study, 14 MAPKKK, one MEK, and two MPK genes were up-regulated by MSP1 (e.g., OSMPK5 gene and MAPKKK1 gene). OSMPK5, also known as $O s B I M K 1$ and $O s M P K 3$, has been reported to be involved in disease resistance responses (Song and Goodman, 2002) and to positively regulate the JA signaling pathway and plant resistance to a chewing herbivore in rice (Wang et al., 2013). Moreover, MAPKKK1 (also known as $O s E D R 1$ and $O s A C D R 1$ ) is a potential positive regulator of fungal disease resistance (Kim et al., 2009a) and a negative regulator of rice bacterial resistance via activation of ethylene biosynthesis (Shen et al., 2011). These results indicate that MAPKs play a vital role in the transduction of MSP1 induced signal to disease resistance in rice.

As a downstream signal of MAPKs, numerous WRKY transcription factors are involved in the development and initiation of stress and defense responses. Among 30 up-regulated genes encoding WRKYs, OsWRKY23, OsWRKY24, OsWRKY28, OsWRKY45, OsWRKY51, OsWRKY53, OsWRKY62, OsWRKY70, OsWRKY71, Os$W R K Y 72$, and $O s W R K Y 76$ have been thoroughly investigated for their roles in rice innate immune responses. Several previous transcriptional analyses showed expression of WRKYs were increased in response to attack by $M$. oryzae (e.g., OsWRKY45, OsWRKY47, OsWRKY53, OsWRKY55, OsWRKY62 and OsWRKY71), and over-expression of some of these genes enhanced resistance to rice blast infection (Bagnaresi et al., 2012; Chujo et al., 2007; Ryu et al., 2006; Shimono et al., 2012; Wei et al., 2013). Moreover, four WRKYs (OsWRKY51, OsWRKY62, OsWRKY71, and OsWRKY76) were reported to be involved in resistance against $X$. oryzae pv. oryzae infection in rice (Hwang et al., 2016; Liu et al., 2016, 2007; Peng et al., 2008). For instance, OsWRKY45 plays important roles in rice resistance to both $M$. oryzae and $X$. oryzae pv. oryzae via SA hormone signaling (Shimono et al., 2012) and negatively modulates resistance against the brown planthopper, Nilaparvata lugens (Huangfu et al., 2016). Moreover, OsWRKY45 and $\mathrm{Pb} 1$ interaction contributes to blast resistance through protection of OsWRKY45 from ubiquitin-proteasome system degradation (Matsushita et al., 2013). These results indicate that complicated signaling of WRKY transcription factors could be initialized by the MSP1 treatment that can be transduced to defense responses.

Phytohormones are central regulators of plant defense. In the present study, the JA, ET, and ABA signaling pathways were significantly upregulated by MSP1 treatment, while only three genes involved in the SA signaling pathway were detected in MSP1 treated samples, which is in accordance with our previous finding that JA and $\mathrm{ABA}$ positively regulate MSP1 induced cell death (Wang et al., 2016). It is well known that JA regulates growth development and stress responses, especially defense responses to herbivores and necrotrophic pathogens (Browse, 2009). Previous studies in rice have revealed that JA signaling was involved in rice basal defense against bacterial and fungal pathogens (Yamada et al., 2012; Yang et al., 2012, 2013). Moreover, ABA was reported to be both a positive and negative regulator of rice disease resistance, depending on the pathogen type and infection stage (Yang et al., 2013). There are also several lines of evidence that suggest ET confers broad-spectrum resistance against the fungal infection (Helliwell et al., 2013). Additionally, numerous studies have shown that SA acts as a positive regulator of immunity against biotrophic pathogens and hemibiotrophic pathogens (Berens et al., 2017; Glazebrook, 2005). 
However, rice plants did not show any increase in SA after inoculation with M. oryzae (Silverman et al., 1995). Limitation of the microarray may be one of the reasons why only three SA signaling genes were identified. Moreover, a recent study revealed that overexpression of MSP1 in rice confers broad-spectrum resistance through modulation of the SA- and JA-mediated signaling pathways (Hong et al., 2017). In addition, a study of the newly identified CP protein, $\mathrm{SsCP} 1$, revealed that the SA signaling pathway is involved in CP-mediated plant defense (Yang et al., 2018). Together, these results indicate that intricate networks of phytohormone signaling are activated by MSP1 treatment, leading to increased rice immunity.

Overall, our study provides a broader picture of the major physiological reactions underlying MSP1-induced PTI signaling at the transcriptional level. Upon treatment with MSP1, elicitor perception and a series of signal transductions (including MAPKs, WRKY transcription factors, hormone signaling and PR proteins) were activated. Our results suggest MSP1 triggers the activation of plant defense responses against various pathogens.

\section{Acknowledgments}

This work was supported by a grant from the Next Generation Bio Green 21 Program (Plant Molecular Breeding Center, PJ008021) and the Basic Science Research Program through the National Research Foundation of Korea (NRF) funded by the Ministry of Education, Science, and Technology (NRF-2018R1A4A1025158), Republic of Korea.

\section{References}

Akamatsu, A., Wong, H. L., Fujiwara, M., Okuda, J., Nishide, K., Uno, K., Imai, K., Umemura, K., Kawasaki, T., Kawano, Y. and Shimamoto, K. 2013. An OsCEBiP/OsCERK1OsRacGEF1-OsRac1 module is an essential early component of chitin-induced rice immunity. Cell Host Microbe. 13:465476.

Antolín-Llovera, M., Ried, M. K., Binder, A. and Parniske, M. 2012. Receptor kinase signaling pathways in plant-microbe interactions. Annu. Rev. Phytopathol. 50:451-473.

Bagnaresi, P., Biselli, C., Orrù, L., Urso, S., Crispino, L., Abbruscato, P., Piffanelli, P., Lupotto, E., Cattivelli, L. and Valè, G. 2012. Comparative transcriptome profiling of the early response to Magnaporthe oryzae in durable resistant vs susceptible rice (Oryza sativa L.) genotypes. PLoS One. 7:e51609.

Berens, M. L., Berry, H. M., Mine, A., Argueso, C. T. and Tsuda, K. 2017. Evolution of hormone signaling networks in plant defense. Annu. Rev. Phytopathol. 55:401-425.
Bilgin, D. D., Zavala, J. A., Zhu, J., Clough, S. J., Ort, D. R. and DeLUCIA, E. H. 2010. Biotic stress globally downregulates photosynthesis genes. Plant Cell Environ. 33:1597-1613.

Boller, T. and Felix, G. 2009. A renaissance of elicitors: perception of microbe-associated molecular patterns and danger signals by pattern-recognition receptors. Annu. Rev. Plant Biol. 60:379-406.

Boller, T. and He, S. Y. 2009. Innate immunity in plants: an arms race between pattern recognition receptors in plants and effectors in microbial pathogens. Science. 324:742-744.

Bolton, M. D. 2009. Primary metabolism and plant defense-fuel for the fire. Mol. Plant. Microbe Interact. 22:487-497.

Boutrot, F. and Zipfel, C. 2017. Function, discovery, and exploitation of plant pattern recognition receptors for broad-spectrum disease resistance. Annu. Rev. Phytopathol. 55:257-286.

Browse, J. 2009. Jasmonate passes muster: a receptor and targets for the defense hormone. Annu. Rev. Plant Biol. 60:183-205.

Chen, K., Fan, B., Du, L. and Chen, Z. 2004. Activation of hypersensitive cell death by pathogen-induced receptor-like protein kinases from Arabidopsis. Plant Mol. Biol. 56:271-283.

Chujo, T., Takai, R., Akimoto-Tomiyama, C., Ando, S., Minami, E., Nagamura, Y., Kaku, H., Shibuya, N., Yasuda, M., Nakashita, H., Umemura, K., Okada, A., Okada, K., Nojiri, H. and Yamane, H. 2007. Involvement of the elicitor-induced gene OsWRKY53 in the expression of defense-related genes in rice. Biochim. Biophys. Acta BBA - Gene Struct. Expr. 1769:497-505.

Dodds, P. N. and Rathjen, J. P. 2010. Plant immunity: towards an integrated view of plant-pathogen interactions. Nat. Rev. Genet. 11:539-548.

Eckardt, N. A. 2011. Induction of phytoalexin biosynthesis: WRKY33 is a target of MAPK signaling. Plant Cell. 23:1190-1190.

Eulgem, T. and Somssich, I. E. 2007. Networks of WRKY transcription factors in defense signaling. Curr. Opin. Plant Biol. 10:366-371.

Gaderer, R., Bonazza, K. and Seidl-Seiboth, V. 2014. Cerato-platanins: a fungal protein family with intriguing properties and application potential. Appl. Microbiol. Biotechnol. 98:47954803.

Glazebrook, J. 2005. Contrasting mechanisms of defense against biotrophic and necrotrophic pathogens. Annu. Rev. Phytopathol. 43:205-227.

Gómez-Gómez, L. and Boller, T. 2000. FLS2: an LRR receptorlike kinase involved in the perception of the bacterial elicitor flagellin in Arabidopsis. Mol. Cell. 5:1003-1011.

Helliwell, E. E., Wang, Q. and Yang, Y. 2013. Transgenic rice with inducible ethylene production exhibits broad-spectrum disease resistance to the fungal pathogens Magnaporthe oryzae and Rhizoctonia solani. Plant Biotechnol. J. 11:33-42.

Hong, Y., Yang, Y., Zhang, H., Huang, L., Li, D. and Song, F. 2017. Overexpression of MoSM1, encoding for an immunityinducing protein from Magnaporthe oryzae, in rice confers broad-spectrum resistance against fungal and bacterial dis- 
eases. Sci. Rep. 7:41037.

Huang, D. W., Sherman, B. T. and Lempicki, R. A. 2008. Systematic and integrative analysis of large gene lists using DAVID bioinformatics resources. Nat. Protoc. 4:44.

Huangfu, J., Li, J., Li, R., Ye, M., Kuai, P., Zhang, T. and Lou, Y. 2016. The transcription factor OsWRKY45 negatively modulates the resistance of rice to the brown planthopper Nilaparvata lugens. Int. J. Mol. Sci. 17:697.

Hwang, S.-H., Kwon, S. I., Jang, J.-Y., Fang, I. L., Lee, H., Choi, C., Park, S., Ahn, I., Bae, S. and Hwang, D.-J. 2016. OsWRKY51, a rice transcription factor, functions as a positive regulator in defense response against Xanthomonas oryzae pv. oryzae. Plant Cell Rep. 35:1975-1985.

Jain, M., Nijhawan, A., Tyagi, A. K. and Khurana, J. P. 2006. Validation of housekeeping genes as internal control for studying gene expression in rice by quantitative real-time PCR. Biochem. Biophys. Res. Commun. 345:646-651.

Jeong, J. S., Mitchell, T. K. and Dean, R. A. 2007. The Magnaporthe grisea snodprot1 homolog, MSP1, is required for virulence. FEMS Microbiol. Lett. 273:157-165.

Jones, J. D. G. and Dangl, J. L. 2006. The plant immune system. Nature. 444:323-329.

Kanehisa, M., Goto, S., Sato, Y., Kawashima, M., Furumichi, M. and Tanabe, M. 2014. Data, information, knowledge and principle: back to metabolism in KEGG. Nucleic Acids Res. 42:D199-D205.

Kangasjärvi, S., Neukermans, J., Li, S., Aro, E.-M. and Noctor, G. 2012. Photosynthesis, photorespiration, and light signalling in defence responses. J. Exp. Bot. 63:1619-1636.

Kim, J.-A., Cho, K., Singh, R., Jung, Y.-H., Jeong, S.-H., Kim, S.-H., Lee, J.-E., Cho, Y.-S., Agrawal, G. K., Rakwal, R., Tamogami, S., Kersten, B., Jeon, J.-S., An, G. and Jwa, N.-S. 2009a. Rice OsACDR1 (Oryza sativa accelerated cell death and resistance 1) is a potential positive regulator of fungal disease resistance. Mol. Cells. 28:431-439.

Kim, S. T., Kang, Y. H., Wang, Y., Wu, J., Park, Z. Y., Rakwal, R., Agrawal, G. K., Lee, S. Y. and Kang, K. Y. 2009b. Secretome analysis of differentially induced proteins in rice suspensioncultured cells triggered by rice blast fungus and elicitor. Proteomics. 9:1302-1313.

Kim, S. T., Kim, S. G., Hwang, D. H., Kang, S. Y., Kim, H. J., Lee, B. H., Lee, J. J. and Kang, K. Y. 2004. Proteomic analysis of pathogen-responsive proteins from rice leaves induced by rice blast fungus, Magnaporthe grisea. Proteomics. 4:3569-3578.

Liu, J., Chen, X., Liang, X., Zhou, X., Yang, F., Liu, J., He, S. Y. and Guo, Z. 2016. Alternative splicing of rice WRKY62 and WRKY76 transcription factor genes in pathogen defense. Plant Physiol. 171:1427-1442.

Liu, L.-J., Zheng, H.-Y., Jiang, F., Guo, W. and Zhou, S.-T. 2014a. Comparative transcriptional analysis of asexual and sexual morphs reveals possible mechanisms in reproductive polyphenism of the cotton aphid. PLoS One. 9:e99506.

Liu, W., Liu, J., Triplett, L., Leach, J. E. and Wang, G.-L. 2014 b.
Novel insights into rice innate immunity against bacterial and fungal pathogens. Annu. Rev. Phytopathol. 52:213-241.

Liu, X., Bai, X., Wang, X. and Chu, C. 2007. OsWRKY71, a rice transcription factor, is involved in rice defense response. $J$. Plant Physiol. 164:969-979.

van Loon, L. C., Rep, M. and Pieterse, C. M. 2006. Significance of inducible defense-related proteins in infected plants. Annu. Rev. Phytopathol. 44:135-162.

Luti, S., Caselli, A., Taiti, C., Bazihizina, N., Gonnelli, C., Mancuso, S. and Pazzagli, L. 2016. PAMP activity of ceratoplatanin during plant interaction: an-Omic Approach. Int. J. Mol. Sci. 17:866.

Major, I. T., Nicole, M.-C., Duplessis, S. and Séguin, A. 2010. Photosynthetic and respiratory changes in leaves of poplar elicited by rust infection. Photosynth. Res. 104:41-48.

Matsushita, A., Inoue, H., Goto, S., Nakayama, A., Sugano, S., Hayashi, N. and Takatsuji, H. 2013. Nuclear ubiquitin proteasome degradation affects WRKY45 function in the rice defense program. Plant J. 73:302-313.

Meng, X. and Zhang, S. 2013. MAPK Cascades in Plant Disease Resistance Signaling. Annu. Rev. Phytopathol. 51:245-266.

Niks, R. E., Qi, X. and Marcel, T. C. 2015. Quantitative resistance to biotrophic filamentous plant pathogens: concepts, misconceptions, and mechanisms. Annu. Rev. Phytopathol. 53:445470.

Pazzagli, L., Seidl-Seiboth, V., Barsottini, M., Vargas, W. A., Scala, A. and Mukherjee, P. K. 2014. Cerato-platanins: elicitors and effectors. Plant Sci. 228:79-87.

Peng, Y., Bartley, L. E., Chen, X., Dardick, C., Chern, M., Ruan, R., Canlas, P. E. and Ronald, P. C. 2008. OsWRKY62 is a negative regulator of basal and Xa21-mediated defense against Xanthomonas oryzae pv. oryzae in rice. Mol. Plant. 1:446-458.

Roberts, M. R. and Paul, N. D. 2006. Seduced by the dark side: integrating molecular and ecological perspectives on the influence of light on plant defence against pests and pathogens. New Phytol. 170:677-699.

Rojas, C. M., Senthil-Kumar, M., Tzin, V. and Mysore, K. S. 2014. Regulation of primary plant metabolism during plantpathogen interactions and its contribution to plant defense. Front. Plant Sci. 5:17.

Ryu, H.-S., Han, M., Lee, S.-K., Cho, J.-I., Ryoo, N., Heu, S., Lee, Y.-H., Bhoo, S. H., Wang, G.-L., Hahn, T.-R. and Jeon, J.-S. 2006. A comprehensive expression analysis of the WRKY gene superfamily in rice plants during defense response. Plant Cell Rep. 25:836-847.

Shah, J., Kachroo, P., Nandi, A. and Klessig, D. F. 2001. A recessive mutation in the Arabidopsis SSI2 gene confers SA- and NPR1-independent expression of PR genes and resistance against bacterial and oomycete pathogens. Plant J. 25:563574.

Shen, X., Liu, H., Yuan, B., Li, X., Xu, C. and Wang, S. 2011. OsEDR1 negatively regulates rice bacterial resistance via activation of ethylene biosynthesis. Plant Cell Environ. 34:179- 
191.

Shimono, M., Koga, H., Akagi, A., Hayashi, N., Goto, S., Sawada, M., Kurihara, T., Matsushita, A., Sugano, S., Jiang, C.-J., Kaku, H., Inoue, H. and Takatsuji, H. 2012. Rice WRKY45 plays important roles in fungal and bacterial disease resistance. Mol. Plant Pathol. 13:83-94.

Shiu, S.-H., Karlowski, W. M., Pan, R., Tzeng, Y.-H., Mayer, K. F. X. and Li, W.-H. 2004. Comparative analysis of the receptor-like kinase family in Arabidopsis and rice. Plant Cell. 16:1220-1234.

Silverman, P., Seskar, M., Kanter, D., Schweizer, P., Metraux, J. P. and Raskin, I. 1995. Salicylic acid in rice (biosynthesis, conjugation, and possible role). Plant Physiol. 108:633-639.

Song, F. and Goodman, R. M. 2002. OsBIMK1, a rice MAP kinase gene involved in disease resistance responses. Planta. 215:997-1005.

Song, W.-Y., Wang, G.-L., Chen, L.-L., Kim, H.-S., Pi, L.-Y., Holsten, T., Gardner, J., Wang, B., Zhai, W.-X., Zhu, L.-H., Fauquet, C. and Ronald, P. 1995. A receptor kinase-like protein encoded by the rice disease resistance gene, Xa21. Science. 270:1804-1806.

Talbot, N. J. 2003. On the trail of a cereal killer: exploring the biology of Magnaporthe grisea. Annu. Rev. Microbiol. 57:177202.

Thimm, O., Bläsing, O., Gibon, Y., Nagel, A., Meyer, S., Krüger, P., Selbig, J., Müller, L. A., Rhee, S. Y. and Stitt, M. 2004. MAPMAN: a user-driven tool to display genomics data sets onto diagrams of metabolic pathways and other biological processes. Plant J. 37:914-939.

Wang, C., Wang, G., Zhang, C., Zhu, P., Dai, H., Yu, N., He, Z., $\mathrm{Xu}, \mathrm{L}$. and Wang, E. 2017. OsCERK1-mediated chitin perception and immune signaling requires receptor-like cytoplasmic kinase 185 to activate an MAPK cascade in rice. Mol. Plant. 10:619-633.

Wang, Q., Li, J., Hu, L., Zhang, T., Zhang, G. and Lou, Y. 2013. OsMPK3 positively regulates the JA signaling pathway and plant resistance to a chewing herbivore in rice. Plant Cell Rep. 32:1075-1084.

Wang, Y., Kwon, S. J., Wu, J., Choi, J., Lee, Y.-H., Agrawal, G. K., Tamogami, S., Rakwal, R., Park, S.-R., Kim, B.-G., Jung, K.-H., Kang, K. Y., Kim, S. G. and Kim, S. T. 2014. Transcriptome analysis of early responsive genes in rice during Magnaporthe oryzae infection. Plant Pathol. J. 30:343-354.
Wang, Y., Wu, J., Kim, S. G., Tsuda, K., Gupta, R., Park, S.Y., Kim, S. T. and Kang, K. Y. 2016. Magnaporthe oryzaesecreted protein MSP1 induces cell death and elicits defense responses in rice. Mol. Plant. Microbe Interact. 29:299-312.

Wei, T., Ou, B., Li, J., Zhao, Y., Guo, D., Zhu, Y., Chen, Z., Gu, H., Li, C., Qin, G. and Qu, L.-J. 2013. Transcriptional profiling of rice early response to Magnaporthe oryzae identified OsWRKYs as important regulators in rice blast resistance. PLoS One. 8:e59720.

Yamada, K., Yamaguchi, K., Shirakawa, T., Nakagami, H., Mine, A., Ishikawa, K., Fujiwara, M., Narusaka, M., Narusaka, Y., Ichimura, K., Kobayashi, Y., Matsui, H., Nomura, Y., Nomoto, M., Tada, Y., Fukao, Y., Fukamizo, T., Tsuda, K., Shirasu, K., Shibuya, N. and Kawasaki, T. 2016. The Arabidopsis CERK1-associated kinase PBL27 connects chitin perception to MAPK activation. EMBO J. 35:2468-2483.

Yamada, S., Kano, A., Tamaoki, D., Miyamoto, A., Shishido, H., Miyoshi, S., Taniguchi, S., Akimitsu, K. and Gomi, K. 2012. Involvement of OsJAZ8 in jasmonate-induced resistance to bacterial blight in rice. Plant Cell Physiol. 53:2060-2072.

Yang, D.-L., Yang, Y. and He, Z. 2013. Roles of plant hormones and their interplay in rice immunity. Mol. Plant. 6:675-685.

Yang, D.-L., Yao, J., Mei, C.-S., Tong, X.-H., Zeng, L.-J., Li, Q., Xiao, L.-T., Sun, T., Li, J., Deng, X.-W., Lee, C. M., Thomashow, M. F., Yang, Y., He, Z. and He, S. Y. 2012. Plant hormone jasmonate prioritizes defense over growth by interfering with gibberellin signaling cascade. Proc. Natl. Acad. Sci. 109:E1192-E1200.

Yang, G., Tang, L., Gong, Y., Xie, J., Fu, Y., Jiang, D., Li, G., Collinge, D. B., Chen, W. and Cheng, J. 2018. A ceratoplatanin protein SsCP1 targets plant PR1 and contributes to virulence of Sclerotinia sclerotiorum. New Phytol. 217:739755.

Yang, Y., Zhang, H., Li, G., Li, W., Wang, X. and Song, F. 2009. Ectopic expression of MgSM1, a Cerato-platanin family protein from Magnaporthe grisea, confers broad-spectrum disease resistance in Arabidopsis. Plant Biotechnol. J. 7:763777.

Zipfel, C., Kunze, G., Chinchilla, D., Caniard, A., Jones, J. D. G., Boller, T. and Felix, G. 2006. Perception of the bacterial PAMP EF-Tu by the receptor EFR restricts Agrobacteriummediated transformation. Cell. 125:749-760. 